\title{
The influence of enclosure design on diurnal activity and stereotypic behaviour in captive Malayan Sun bears (Helarctos malayanus)
}

\begin{abstract}
The effect of enclosure design on diurnal activity and stereotypic behaviour was assessed in 17 adult Malayan Sun bears (Helarctos malayanus), kept either in barren indoor enclosures or relatively enriched outdoor enclosures. Locomotion was the most frequent activity observed in the indoor bears, followed by resting. In contrast, conspecifics housed outdoors spent most of the time resting. Eleven forms of stereotypic behaviours were recorded in the bears, with pacing being the most common. The frequency and repertoire of stereotypies were significantly higher in the indoor bears irrespective of enclosure size. Novel forms of locomotor (forward-reverse pacing) and oral (allo-sucking) stereotypies were recorded. Oral stereotypies were predominant in the bears housed indoors, while patrolling was confined to the outdoor bears. Enclosure complexity significantly influences activity budget and occurrence of stereotypic behaviours, highlighting the importance of appropriate enclosure design and enrichment for the welfare of captive bears.
\end{abstract}

Keyword: Activity budget; Enclosure design; Helarctos malayanus; Malayan Sun bear; Stereotypic behaviour; Welfare 